\title{
Entretien avec Mohammed El Amraoui
}

Interview with Mohammed El Amraoui

Mohammed El Amraoui

\section{CpenEdition}

Journals

Édition électronique

URL : http://journals.openedition.org/recherchestravaux/1799

DOI : 10.4000/recherchestravaux.1799

ISSN : 1969-6434

Éditeur

UGA Éditions/Université Grenoble Alpes

Édition imprimée

ISBN : 978-2-37747-165-2

ISSN : 0151-1874

Référence électronique

Mohammed El Amraoui, «Entretien avec Mohammed El Amraoui », Recherches \& Travaux [En ligne],

95 | 2019, mis en ligne le 05 décembre 2019, consulté le 08 septembre 2020. URL : http://

journals.openedition.org/recherchestravaux/1799; DOI : https://doi.org/10.4000/recherchestravaux. 1799

Ce document a été généré automatiquement le 8 septembre 2020

(c) Recherches \& Travaux 


\title{
Entretien avec Mohammed El Amraoui
}

\author{
Interview with Mohammed El Amraoui
}

Mohammed El Amraoui

\section{NOTE DE L'ÉDITEUR}

Cet entretien est une version retranscrite puis remaniée par Aline Marchand de deux échanges qui se sont tenus le 9 novembre 2017 à Grenoble, puis revue par Mohammed El Amraoui : un entretien mené par Camille Eynard, Gaëlle Grella, Gaëlle Le Port, Kevin Woringer, étudiants de Master (parcours « Littérature, critique et création ») à l'université de Grenoble, en ouverture de la première session du colloque «L'écrivaintraducteur : ethos et style d'un co-auteur ». L'intégralité de l'entretien, enregistré par Kevin Woringer, est en ligne : <https://archive.org/details/ElAmraoui>. L'entretien est suivi de la lecture de deux textes par l'auteur (les trois dernières minutes) : «Présence massive... » (De ce côté-ci et alentour, Lié / Chaillé-sous-les-Ormeaux, L'Idée bleue, coll. « Le dé bleu », 2006, p. 80-81) et « H. » (Récits, partitions et photographies : poésie, Genouilleux, La Passe du vent, 2007, p. 9-13). La version ici retranscrite est également enrichie des propos de Mohammed El Amraoui lors de la table-ronde animée par Pascale Roux, le même jour, au Café des Arts, dont une captation est disponible en ligne : <https://archive.org/details/ElAmraouiGharrafiJeghamCafDesArtsV2>.

Question: Lors de lectures publiques de vos poèmes parus dans des éditions bilingues, vous commencez souvent par les lire en arabe, puis en français : quelle relation entretenezvous avec ces deux langues?

Mohammed El Amraoui : Dans ma trajectoire personnelle, j'ai d'abord écrit en arabe. Je suis arrivé en France à l'âge de 24 ans. J'ai commencé à écrire d'abord en arabe classique parce que la poésie au Maroc, ainsi que dans le monde arabe, était très présente ; ça l'est toujours, mais le public fréquente moins les salles de lecture peutêtre aujourd'hui. Les poètes, jeunes et moins jeunes, publiaient dans les revues et 
dans les journaux, dans les quotidiens ; cela continue encore aujourd'hui. On écrivait souvent par imitation, tant la poésie contemporaine était populaire. On lisait les poèmes dans les MJC, l'université et dans d'autres lieux ; un peu comme les slameurs aujourd'hui. À l'école, j'étais assez faible en français jusqu'à la seconde, j'ai commencé alors à écrire de la poésie dans cette langue juste pour pouvoir mieux intégrer quelques mots, et quelques structures syntaxiques aussi. C'est devenu plus sérieux quand j'ai commencé à écouter des poètes et surtout des comédiens lire de la poésie. J'allais emprunter des cassettes audio au centre culturel de Fès, et je les écoutais tous les jours. L'écriture en français est donc arrivée plus tard. Quant à la traduction de l'arabe en français, c'est surtout ici que sa nécessité est survenue : quand on lit en public de la poésie en arabe, il faut bien que les gens comprennent, si bien que la traduction s'est imposée par elle-même. D'un autre côté, quand j'écris en arabe je ne pense pas en français, et inversement, quand j'écris en français je ne pense pas en arabe, c'est-à-dire que j'écris dans la langue, dans sa singularité. La difficulté vient parfois de là : on joue avec les mots, avec les signifiants sonores, avec les répétitions, avec de nombreux éléments que l'on ne retrouve pas facilement quand on passe dans l'autre langue. Je n'écris donc pas d'abord en arabe, et après en français - ce qui relèverait de la traduction; en revanche, il m'arrive d'écrire en parallèle, au cours d'une même journée, dans les deux langues - on ne sait d'ailleurs pas pourquoi ni comment se passe le processus du passage d'une langue à l'autre.

Q. : Vous avez, vous-même, traduit certains de vos recueils en particulier celui qui s'intitule Des moineaux dans la tête عصافير في الرأس 1 : comment concevez-vous cette pratique de l'autotraduction?

Mohammed El Amraoui : C'est plus pratique et cela permet aussi de gagner du temps : j'ai écrit le texte - on peut dire que j'ai acquis une connaissance du français, je peux donc le traduire rapidement, mais cela reste toujours un premier jet. Et c'est justement le risque : comme on est assez proche de ce que l'on a écrit, on peut passer à côté d'une deuxième interprétation, ne pas voir quelque chose, trouver évidente une phrase qui ne l'est pas pour une personne extérieure qui connaît les deux langues - j'en ai fait l'expérience avec Catherine Charruau, qui traduit surtout des romans chez Actes Sud, et qui s'étonne parfois de mes autotraductions. On a toujours besoin de recul, de distance, d'un autre regard. Cela m'est aussi arrivé d'être traduit par d'autres dès le premier jet, et dans ce cas-là je relis.

Quand j'écris, je ne pense alors pas du tout à la traduction, car je m'installe dans les spécificités de la langue dans laquelle j'écris. Et quand j'écris dans la langue, je ne suis pas que dans le sens, mais aussi dans la forme, à tel point que quelques fois on affronte l'intraduisible. C'est au moment de la traduction que les difficultés se révèlent, devant un travail sur les sonorités par exemple et ce qui relève de l'intraduisible radical. Toute l'écriture peut reposer, dans quelques cas, sur le signifiant sonore ou graphique et pas seulement sur le niveau sémantique. Par exemple, dans un texte qui s'intitule « $E x^{2} »$, que j'ai écrit en français, tous les mots commencent par ce préfixe-là, c'est-à-dire « ex », qui signifie " mettre dehors ou audelà ", et qui a donné des mots ayant cette signification du rejet ou du surplus : expulsion, exil, excrément, extérieur, excès, extraordinaire - à chaque fois il y a ce sens de l'extériorisation ou de l'expulsion. Tout le sens du poème est basé sur cette répétition de ex- sans cesse du début jusqu'à la fin. Et donc sur la forme et non pas 
seulement sur le contenu. Dans la traduction, cela, qui est essentiel dans la construction du poème, disparaîtrait.

Inversement, une seule fois, une expérience bilingue s'est imposée à moi, dans un texte sonore qui s'appelle «Hajar" / حجر / Pierres" où le français et l'arabe s'imbriquaient : le mot hajar, est basé sur la racine tri-consonantique (comme tous les mots arabes, mis à part les emprunts), $h, j, r$. Quand on fait des permutations sur ces trois lettres/sons ou quand on change les voyelles, cela donne plusieurs mots qui se ressemblent au niveau sonore : hajar/pierres, hijr/giron, jurh/blessure, juhr/repaire ou terrier, haraj/trouble ou gêne..., qui vont à peu près dans le même sens que prend le poème; ce qui disparaît complètement en français quand on le traduit. Ces mots ponctuent donc en arabe la lecture du poème en français. C'est toujours problématique, qu'il s'agisse de mon propre texte ou de celui de quelqu'un d'autre, cela revient toujours à la question du passage difficile d'une langue à l'autre.

Q. : Vous avez traduit, notamment, de nombreux poèmes palestiniens et marocains sous la forme d'anthologies ${ }^{3}$. Votre expérience de l'autotraduction se distingue-t-elle de cette traduction d'autres poètes?

Mohammed El Amraoui : Un ami que j'ai traduit, le poète palestinien Anas Alaili ${ }^{4}$, qui écrit de très beaux poèmes, très brefs et intelligents, de petites narrations, s'intéresse plus au sens qu'au travail formel sur les sonorités de la langue, il n'y a pas de surcharge métaphorique ou rythmique. Il est arrivé des fois que la traduction révèle une petite faiblesse dans la construction ou dans une image qui peut paraître contradictoire, alors il réécrit son texte à partir de la traduction parce qu'il veut que son texte passe dans l'autre langue, il ne veut pas se permettre des fragilités qui, en arabe, peuvent passer presque inaperçues. C'est la traduction qui, quelques fois, les révèle. Et la question du sens se pose, quoi qu'on dise, qu'il s'agisse d'un travail sur la forme ou que le poète puisse se permettre le non-sens; la question se posera toujours. Car la traduction, c'est faire passer quelque chose. C'est la question de la justification - intérieure au poème - de l'utilisation d'un mot par exemple. Même dans la traduction littérale : pour حزين (hazin), par exemple, c'est un choix subjectif de le rendre par le mot triste et non pas par mélancolique ou encore par un autre synonyme. On peut respecter la syntaxe, la place du mot, on peut volontairement prendre moins de liberté, mais on choisit tout de même un mot parmi des synonymes. La traduction est donc liée à notre conception et à notre expérience dans l'écriture. Je viens de faire l'expérience de traduire un texte deux ans après: au moment de la parution de l'anthologie de poètes palestiniens que j'avais traduits, j'ai voulu relire et j'ai réécrit un nombre de phrases incroyable! Avec le temps, on voit les choses autrement. On évolue dans l'écriture et donc on évolue aussi dans la traduction et ainsi de suite ; les deux sont liées.

Quand on se traduit soi-même, on reste proche de ce qu'on voulait faire passer dans le poème comme image ou émotion. Quant à la traduction des autres poètes, on est surtout en face de mots et de phrases, et on essaye de trouver une fenêtre ou une porte pour accéder à l'univers qu'ils créent. Quand on connait le poète, on peut lui poser des questions, sinon on cherche.

Q. : Quelles sont, pour vous, les principales difficultés dans la traduction - vous parliez $\mathrm{d}^{\prime} \ll$ intraduisible radical »?

Mohammed El Amraoui : Il arrive que des images paraissent surréalistes ou un peu bizarres en français, alors qu'en arabe non, parce qu'elles font allusion soit au Coran, 
soit à la poésie préislamique, soit à un passage de Gilgamesh; en tout cas, à une référence spécifique que le traducteur devrait connaître d'une manière ou d'une autre.

La question du genre est une autre difficulté : par exemple, dans un court poème, un poète a écrit un poème où il parlait à l'arbre - qui est féminin en arabe - comme s'il parlait à une femme. Je cherchais un arbre qui soit féminin dans la langue française : je n'ai toujours pas trouvé! Les plantes oui, mais pas les arbres. Alors il faut trouver des moyens de détournement ou d'ajout pour rendre possible le passage. On a beau se dire qu'on peut prendre cette liberté, il reste néanmoins une composante essentielle, pas seulement poétique, qui est celle de la langue, dans sa singularité, parce que tous les poètes cherchent ce que la langue peut leur donner comme possibilités, cherchent comment réinventer la langue et créer la leur propre. Cela a déjà été dit, mais c'est bien de le rappeler. Ainsi au problème de la langue s'ajoute la langue du poète luimême, enrichie d'autres langues, surtout depuis que la traduction existe. Depuis son apparition, les poètes n'écrivent pas de la même manière : depuis la découverte des traductions de Rítsos, de Rimbaud ou de Carver, nos références ne se limitent plus à la poésie préislamique, islamique, abbasside ou andalouse; ce qui ajoute un autre niveau de complexité.

Q. : Le répertoire culturel semble très important pour le traducteur que vous êtes, cet héritage occupe-t-il la même place dans votre pratique de l'écriture? Vous avez suivi des études en linguistique : quel en a été l'impact dans la genèse de vos poèmes ?

Mohammed El Amraoui : La formation (en dehors de la pratique de l'écriture) peut avoir un impact sur ce qu'on écrit. Si l'on fait de la médecine, il est possible que notre bagage, que notre savoir, puisse s'intégrer dans notre écriture littéraire, pourquoi pas. Quand on fait de la linguistique, c'est sûr, on s'interroge sur la langue. Mon itinéraire : j'ai fait des études de linguistique, j'étais alors au Maroc, au département de la langue française, mais je travaillais déjà (et j'ai continué plus tard ici à travailler) sur un long texte ancien du XIII ${ }^{e}$ siècle, écrit en arabe par un linguiste persan qui s'appelle as-Sakkaki, مفتاح العلوم [La Clef des sciences (du langage) ${ }^{5}$ : il proposait déjà une conception globale du système linguistique et considérait la langue dans ses différentes facettes (de la phonétique jusqu'à la sémantique). Moi je travaillais surtout sur le chapitre qui concerne la sémantique, et c'est la question du sens, et de la forme, qui me troublait déjà dans la langue elle-même, c'est-à-dire en arabe.

Après, lorsqu'on passe à la traduction, que ce soit de la terminologie ou des exemples cités (qui sont dans le cas de ce livre et des ouvrages anciens soit tirés de la langue ordinaire, celle de la communication entre les gens, soit du Coran, soit de la poésie), on est face à nombre de complexités. Chez ce linguiste, toutes les citations littéraires ou religieuses reçoivent le même traitement qu'un énoncé ordinaire, et l'énoncé ordinaire va lui-même avoir un statut littéraire, d'une certaine manière, c'est-à-dire par exemple que lorsqu'il cite le Coran, il le met sur le même plan de l'analyse linguistique, qu'un vers d'un poète ou un énoncé quelconque, même si le verset coranique a plus de légitimité pour affirmer ou infirmer une règle grammaticale ou sémantique.

La question de l'écriture, de la langue, du traitement littéraire était déjà là dès le départ. On traduisait cela chez les Arabes par la rhétorique, cela correspond plus à ce qu'on appelle aujourd'hui en linguistique, la pragmatique ou les théories de 
l'énonciation qui travaillent beaucoup sur la langue non seulement écrite mais surtout parlée. C'était troublant, complexe, ça l'est toujours pour moi, parce qu'il cite par exemple le vers d'un poète mais il le traite comme si c'était de la parole énoncée dans un contexte bien défini, alors que la pragmatique linguistique contemporaine fait la différence entre la parole et l'écrit. Dans l'histoire occidentale (de manière générale), il y a une distinction entre l'édifice rhétorique qui permet à un orateur de parler, d'argumenter ou d'émouvoir et la poétique depuis Aristote. Dans celle d'asSakkaki et les théoriciens qui ont travaillé sur l'arabe, cette distinction n'existe pas vraiment : le poème, la phrase sacrée et la phrase ordinaire sont liés et se donnent dans l'analyse comme des énoncés produisant un sens selon des exigences contextuelles. Tout cela me poussait à m'interroger quand je lisais des œuvres littéraires. Les questions n'étaient pas seulement liées à mon parcours universitaire mais à mon quotidien - c'était aussi un souci : quand on écrit, tout ce qu'on lit sur, autour, tout ce qui est métalangage, vient nous interroger dans nos lectures, dans notre pratique. Quand je pratiquais l'écriture, c'était pareil, l'impact était là. J'écris dans une langue arabe classique qui n'est pas vraiment parlée puisqu'elle est différente de l'arabe marocain qui est ma langue maternelle. Autrement dit, j'écris le réel et l'imaginaire dans une langue que je ne parle pas quotidiennement.

Un autre étage de complexité : l'écriture, surtout l'acte littéraire, est à la fois miconsciente mi-inconsciente. On est à mi-chemin entre le mot tel qu'il est en lui-même et la perception qu'il va générer, et puis la perception des choses qui viennent de l'extérieur. Je veux parler de quelque chose ou je veux juste que les sonorités et les mots forment quelque chose. Là, je veux dire que mon bagage n'intervient pas forcément de façon consciente parce que j'écris, je n'analyse pas, je ne fais pas le commentaire. Mais je sais que ce que je sais avant, sur la langue, va être là quelque part. Quand on fait une rature, quand on choisit un mot ou un autre, ce n'est pas de l'écriture automatique, ce n'est pas complètement inconscient, c'est-à-dire j'analyse en même temps, mais j'analyse avec une composante intuitive beaucoup plus grande que si je dois reformuler une phrase d'un essai par exemple. Si vous me dites «Vous allez parler de la poésie ", alors ce serait parler de, alors que quand j'écris de la poésie, je ne parle pas de, je suis dedans. L'analyse se fait dans cette composante intuitive. Entre mon bagage, mon savoir et l'écriture, il existe donc quand même des ponts, mais on ne sait pas comment on les traverse, en vaisseau, en voiture, on prend des risques, mais ce qui est sûr c'est qu'il faut presque s'interdire le rationnel parce que sinon on fait dans le commentaire. Surtout qu'une grande part de la poésie puise dans le mystère, d'une certaine manière, et quand je dis "mystère ", c'est le mystère $\mathrm{du}$ réel aussi, de tout ce qui vient vers nous, on l'interroge et on ne comprend pas toujours ce qui se passe, en nous et à l'extérieur. Je parle de la poésie de façon générale, non seulement métaphorique, mais aussi la plus simple, même celle de Jacques Prévert qui est parfois très simple, même les poètes américains qui sont dans la narration, on trouve dans leur texte aussi une part de mystère incroyable. Et on essaie de la «traduire » en mots, mais des fois ce sont les mots qui prennent un peu le dessus, ils ont leur propre logique, ils génèrent quelque chose qui nous dépasse, quelques fois on comprend, on saisit le sens bien plus tard de ce qu'on a écrit. On écrit, on sait qu'il y a quelque chose, mais je ne sais pas pourquoi cette association de mots, cette image-là est venue, elle s'impose à moi, je ne suis pas capable tout de suite de l'analyser parce que je n'ai pas envie, parce que je sens qu'il y a quelque chose dedans, et je laisse fermenter, et puis des fois je me trompe, je me dis que cela ne veut 
rien dire, que c'est n'importe quoi, des fois je me dis « Ça y est, j'ai compris le rapport entre ce fragment et ce fragment ».

Q. : En parcourant vos recueils, on perçoit à quel point la forme poétique vous importe aussi...

Mohammed El Amraoui : La question de la forme est en lien surtout avec la pratique littéraire dans une langue spécifique. J'écris dans les sonorités de la langue, dans ses expressions, dans tout ce que je sais un petit peu et des fois je rencontre aussi des formules dans mes lectures que je vais après peut-être reprendre à ma manière dans la poésie. La question de la forme vient donc de la langue. En revanche, la forme de la page, la mise en page, l'éclatement des mots sur la page, tout cela vient un petit peu d'une certaine fascination lors de mes lectures: c'est cette liberté que les poètes contemporains ont commencé à avoir pour créer leur propre rythme. Moi, par exemple, quand je mets un seul mot dans une phrase, cela veut dire qu'il est très important, qu'au niveau de la diction je dois faire une pause, je le laisse presque en suspens, je fais le silence entre avant et après. Des fois, surtout dans ce recueil-là De ce côté-ci et alentours ${ }^{6}$, c'est une masse, c'est tout un corps de mots, c'est plus saccadé... Mais en fait, là, sur cette question de la forme, ce n'est pas mon apprentissage linguistique et universitaire qui agit, mais ce sont surtout mes lectures de la poésie contemporaine, laquelle, à un certain moment, s'est imposée à moi : la forme classique n'est pas capable de répondre à mon urgence intérieure, c'est un moule rigide; les grands poètes classiques ont fait des variations sur la langue mais ils ne pouvaient pas aller plus loin. Maintenant, avec la poésie contemporaine, on a une grande liberté, mais aussi une grande difficulté : comment être libre, et en même temps justifier ton choix et ne pas faire n'importe quoi non plus?

Q. : Quelle relation établissez-vous entre vos poèmes et leurs illustrations ? On songe par exemple à votre collaboration avec Fanny Batt.

Mohammed El Amraoui : C'était très simple en fait, j'avais écrit les poèmes et j'ai donné à Fanny Batt pour voir ce qu'elle pouvait faire avec. Des fois, c'est le poème luimême dans sa globalité qui a donné le geste, et des fois Fanny ne s'intéressait qu'à une parcelle du poème, et comme elle n'est pas dans le figuratif mais dans la couleur, elle percevait les couleurs et le mouvement du poème. On voit des traits, des lignes complexes, des formes et silhouettes. Il s'agit plus d'un dialogue entre deux espaces artistiques, que d'une illustration. Ce recueil (Des moineaux dans la tête/الرافير في contient des poèmes écrits en arabe à différentes époques. Il y a plusieurs (الرأس فير styles dans trois chapitres. Leur cohérence vient du fait que c'est un seul auteur qui les a écrits. Un seul auteur empli de plusieurs voix. Et cette sorte de polyphonie est unifiée par la peinture qui, elle, est cohérente, tantôt lumineuse, tantôt sombre, son style est toujours reconnaissable. On voulait faire un livre à trois dimensions : deux langues en vis-à-vis - l'une traduit l'autre - et une troisième qui fait le lien et qui est la peinture. Mais généralement, quand on travaille avec un plasticien, on lui donne vraiment carte blanche, on sait ce qu'il fait, on aime ce qu'il fait, alors on lui dit «Prends ta liberté », on ne va pas lui expliquer le texte.

Q. : Vous parliez de rythme. La musique fait-elle intrinsèquement partie de votre écriture poétique?

Mohammed El Amraoui : J'ai toujours été lié à la musique. Je fais un peu de la percussion et la musicalité dans le poème est très importante pour moi. J'écris avec la voix, qui traverse toujours le poème. On a parlé tout à l'heure de l'importance de la 
mise en page, de l'éclatement des mots sur la page qui peut correspondre quelques fois à ces arrêts, ces silences qui font partie aussi de la musique. La musique, ce n'est pas forcément l'harmonie, ce n'est pas forcément la continuité des notes qui va créer quelque chose de très harmonieux ; les ruptures, comme on le voit beaucoup dans la musique contemporaine, et les silences, en font partie aussi. La musicalité propre à la langue se retrouve dans la poésie, qui essaie aussi un peu de de la réinventer et qui est déjà intrinsèque à la langue. En revanche, quand on travaille avec des musiciens qui, eux, travaillent sur des instruments, c'est un va-et-vient, basé sur des affinités, c'est un dialogue. On n'est jamais dans l'illustration, on est à ce moment-là, quand on va sur scène par exemple, dans un espace où la musique va faire partie du spectacle, et la poésie aussi ; ce sont deux composantes liées entre elles par le désir de créer un nouvel espace. Et cela même si les musiciens demandent toujours ce que je veux comme musique, donnant à la poésie une place plus privilégiée. Dans ce dialogue, on essaie d'instaurer quelque chose de commun, un troisième élément. J'ai toujours aimé travailler en rapport avec d'autres champs artistiques, on se sent moins seul. Et des fois, le musicien (le plasticien aussi) peut interroger notre propre travail et par làmême nous fait évoluer, on n'écrit pas après de la même manière. J'ai travaillé beaucoup avec des musiciens de jazz (avec le Trio Zyriab), de la dub (avec Brain Damage ${ }^{8}$ ), de la musique classique, de la musique marocaine ${ }^{9}$... Mon écriture a évolué ensuite, ou a avancé, pas au sens de progrès - celui-ci existe en science, mais pas en écriture. On peut évoluer d'une étape à l'autre chronologiquement, mais certains moments d'écriture sont des moments de grâce vers lesquels on a de la peine quelques fois à revenir. Et les musiciens m'apportaient parfois ce cadeau : retrouver ces quelques moments ; des fois juste après la répétition, même le soir, j'écrivais.

Q. : La musique permet-elle également de fédérer un public plus large autour de la poésie?

Mohammed El Amraoui : La question du public ne se pose pas au début, non. C'est d'abord des affinités, des rencontres. On rencontre un musicien dans un festival de poésie, par exemple, on improvise, et l'idée germe de monter un projet d'une collaboration. J'ai fait un CD qui s'appelle Tessons ${ }^{10}$ avec deux musiciens, Antoine Birot et Maurice Spitz. J'étais en résidence d'écriture au Pays du Layon, près de Rochefortsur-Loire, et on m'a proposé un jour d'être accompagné par Antoine, qui jouait du ney (une flûte en roseau, souvent présente dans la musique turque, arabe et persane) et on a improvisé : on ne s'est vus même pas une demi-heure pour faire connaissance, essayer quelques sons, et un jour il m'a appelé pour me proposer de monter quelque chose avec un autre musicien contrebassiste. Voilà comment c'est venu. On a donné après des spectacles puis on a fait un $\mathrm{CD}$. Ce sont donc des affinités, des dialogues entre un champ artistique et un autre, dans la mesure où ce que j'écris est souvent destiné non seulement à la page mais à la voix. Tout le corps compte, les cordes vocales sont impliquées : ce travail est déjà musical, et la seule difficulté justement c'est d'éviter que la musique n'étouffe ni n'embellisse trop, pour conserver un va-etvient, un dialogue, et non l'annexion de l'un par l'autre.

Q. : Dans Récits, partitions et photographies ${ }^{11}$, vous évoquez des «lignes intérieures ${ }^{12}$ ». Pour le poète et pour son traducteur, la recherche d'une même image dans une langue et dans une autre est-elle issue de ces mêmes lignes intérieures?

Mohammed El Amraoui : Avec des nuances. Oui, dans le sens de ce qui permet la traduction, le passage d'un sens à l'autre, d'un poème à l'autre, d'une langue à l'autre, c'est une dimension presque universelle dans le parcours, dans la recherche, de ce 
qui est proprement humain ; les "lignes intérieures " sont à peu près les mêmes en tant que concept, je veux dire en tant que représentation mentale, en tant qu'image dans la tête. Mais cette image se transforme une fois qu'elle devient mots dans la langue. Un mot peut provoquer une image dans la tête. L'image, je la conçois. Quand je lui donne après une présence dans la langue, alors là tout à coup, quelque chose se transforme en elle et en moi. Par exemple, quand on est petit, en langue arabe, on apprend le féminin et le masculin, et le mot «arbre » qui se dit شجرة /shajara est féminin. On a donc presque l'impression que l'arbre est naturellement féminin, parce que la langue lui a donné cette singularité, et on se rend compte après, qu'en français, il est masculin, que le féminin de ce mot n'est pas universel, que l'arbre en tant que concept est neutre. Ces lignes intérieures peuvent donc prendre une forme particulière et singulière dans la langue, dans ma représentation linguistique. Après, elle prend encore une forme plus particulière dans ma langue à moi de poète; je dis "ma langue de poète " parce que le poète essaie dans la langue de recréer la langue, d'inventer sa propre langue, c'est vraiment son idéal, son but ultime.

Je parlais tout à l'heure de ces moments de grâce qu'on voudrait retrouver et dans lesquels on touche cette possibilité de trouver sa propre langue. Les «lignes intérieures » sont donc là, dans l'une et l'autre langue, mais on ne les traverse pas de la même manière, c'est pourquoi j'ai nuancé : tout dépend de la langue elle-même, de la langue du poète et de son parcours. Et quand je dis «intérieur ", je veux dire ma langue à moi, mon français à moi, mon arabe à moi, et la poésie donne cette possibilité - la narration aussi mais surtout au moment où le narrateur commence à travailler sa langue aussi, sa formulation et pas seulement le sens. On a tous des " lignes intérieures » qui sont parfois obliques et qui se chevauchent entre elles, qui s'entrecroisent. Je parlais de la complexité, j'aime bien ce mot-là, c'est le philosophe Edgar Morin qui parle de la pensée complexe ${ }^{13}$, et la poésie comporte toute cette complexité, entre sa propre langue et la langue dans laquelle il écrit (c'est-à-dire la langue de tout le monde), entre le sens et la forme, entre le sens qui m'échappe et le sens que je voudrais donner, et ces lignes-là. Le poète est au-dessus du fil qui relie ces éléments comme un funambule, qui prend des risques, qui est là, qui tâtonne, qui ne doit pas tomber, qui doit rester en équilibre. Cet équilibre-là, qui est celui de mon sens et du sens propre au poème, c'est-à-dire celui qui m'échappe, qui est dans la logique du poème lui-même, que je retrouve parfois bien plus tard, a posteriori.

\section{Q. : C'est donc une tension qui caractérise le langage poétique?}

Mohammed El Amraoui : Oui, j'aime bien le mot «tension ». C'est une tension entre ce qui est dans ma tête, dans mes sensations et ce que je veux donner. Des fois, le poème peut presque paraître clair, quotidien, il parle de petites choses, et le mystère est dans ce quelque chose dont je parle. Mais parfois il peut être comme dans un rêve, des bribes de choses qui viennent à nous, qui nous traversent, qui se livrent à nous avec les mots. On est entre ces deux-là, c'est une tension entre ce qui est en moi et ce qui après devient mot sur la page ou dans la voix.

Q. : Lorsque vous comprenez autrement un poème en le relisant plus tard, songez-vous alors qu'il faudrait le réécrire, que vous vous êtes trompé au moment de son écriture?

Mohammed El Amraoui : Non, ce n'est pas qu'on se trompe, simplement certaines choses nous échappent dans la structure elle-même. Comme je le disais plus tôt, parfois on est dans les sonorités et les mots qui viennent, on a surtout envie d'écrire sans savoir quoi, d'autres fois, non, un propos préexiste. Un jour, je voulais parler de 
ce rapport entre le silence et la voix. Comme je parle beaucoup, j'ai commencé par écrire quelques phrases sur cette question de parler ou ne pas parler, de dire ou ne pas dire. Ce poème, dans De ce côté-ci alentour, commence par: "Je dis ainsi quelqu'un parle, je dis ainsi quelqu'un parle à ma place, je dis ainsi quelqu'un parle à ma place quand je parle, et qu'en moi, c'est du silence que je veux émettre ${ }^{14}$ ». Tout a commencé ainsi : vouloir interroger ce moment où on parle et où on a l'impression que c'est quelqu'un d'autre qui parle à notre place. On a l'impression qu'on a un masque, que ce n'est pas tout à fait nous. Ainsi j'ai l'impression que quelqu'un d'autre parle à ma place, le moment où je parle alors que moi finalement j'ai envie de me mettre en silence, et je n'y arrive pas. Et à la fin du poème, je parle des «syllabes, syllabe après syllabe, tranches fines, c'est ce qui reste ${ }^{15}$ ", ce qui m'a un peu échappé. Je travaillais le poème et je butais contre ça et après je me suis dit que non, il faut arrêter le poème là, ce n'est pas la peine d'aller plus loin, c'était juste une intuition. Après je me suis dit que oui, au début, je parle et je m'entends parler alors que j'ai envie de me taire, après il ne reste que des syllabes, les syllabes ne donnent pas forcément du sens, ce n'est pas un mot, le mot peut être une syllabe mais la syllabe elle-même ne produit pas vraiment de sens, c'est donc comme des tranches qui restent. Et entre les deux, c'est la question de la parole, du silence, de la nuit, du mystère, de l'indicible, de la langue elle-même. Cette fin-là m'a un peu échappé, il fallait la laisser suspendue, mais après j'ai compris qu'il y avait une logique, une logique qui parfois nous échappe. Et c'est bien aussi, sinon on reste dans l'analyse, dans le commentaire, dans le rationnel. Or l'essentiel de notre quotidien n'est pas tout à fait rationnel, déjà la nuit avec le rêve, ou notre tentative d'articuler nos sensations dans la communication avec les autres... Et si on prête vraiment attention à ce qui se passe entre un œil et un œil, entre un regard et un regard, entre un geste et un geste, etc., on découvre que c'est assez mystérieux, très compliqué et difficile ; cela nous échappe tout le temps. C'est ce qui fait la richesse de l'humain. Et la poésie le prend en charge d'une certaine manière, elle essaie d'intégrer cette grande composante qui est dans notre quotidien, qui apparait comme moindre, parce qu'on est dans le calcul, dans l'horloge, parce qu'on essaie d'organiser le temps pour ne pas devenir fou. Mais, tout le monde le sait, une grande part du mystérieux s'immisce dans notre quotidien lui-même. Lors de nombreuses conversations avec des amis, ces derniers me disaient « moi j'aime bien écrire sur des choses simples, le quotidien », et moi je dis : les choses simples ne sont jamais simples, je peux rencontrer, en même temps que je prends mon café, plein de choses métaphysiques, des questionnements sur la mort et la vie. L'étrange, l'absurde et le mystère traversent notre quotidien. J'utilise les mots " étrange » et «mystère » dans leur sens propre, sans leur donner une dimension faussement mystique ou ésotérique. Je dis bien "mystique fausse ou artificielle ». La mystique authentique ne me dérange pas. Ça revient à ce que je disais tout à l'heure, il existe toujours une tension entre dire et ne pas dire, désirer dire et ce qui échappe. 


\section{NOTES}

1. M. El Amraoui, 2016, Des moineaux dans la tête/traduit par l'auteur et عصافير في الرأس, Catherine Charruau, Lyon, Jacques André.

2. M. El Amraoui, 2013, Ex. suivi de Pierres-Hajar (livre-CD), Fidel Anthelme X. Des extraits sont consultables en ligne, sur le site de l'auteur : <https://www.mohammedelamraoui.com/ex-suivide-hajar-hjr>. Plusieurs interprétations de ce poème sont visibles sur le web, parmi lesquelles <https://www.youtube.com/watch?reload=9\&v=TwTLMYrSS08>.

3. Anthologie de la poésie marocaine contemporaine (poèmes choisis, présentés et traduits par $\mathrm{M}$. El Amraoui avec C. Charruau); Bacchanales (Revue de la Maison de la poésie Rhône-Alpes), $\mathrm{n}^{\circ} 38$, mars 2006 ; Interludes poétiques de Palestine, M. El Amraoui (trad.), Le temps des cerises et Maison de la poésie Rhône-Alpes, 2019.

4. A. Alaili, Avec une petite différence, Toucy /Châteauroux-les-Alpes, Polder, 2010 (مع فارق بسيط, et Étreintes tardives .Paris, L'Harmattan, عناقات متأخرة, 2016 /

5. Al-Sakkaki (ou as-Sakkaki السكاكي est l'un des plus importants rhétoriciens de langue arabe, né en 1160 dans le Khwarezm et mort en 1229, à Ferghana. Son ouvrage La Clé des sciences (Miftâh al-'Ulûm), est la référence sur laquelle se fondent la classification et le système d'al-balâgha (rhétorique arabe). Il est constitué de plusieurs chapitres commençant par la phonétique ou la phonologie jusqu'à la logique, en passant par la grammaire, la sémantique-pragmatique ou la théorie de l'énonciation et les figures de style.

6. M. El Amraoui, De ce côté-ci et alentours, ouvr. cité.

7. Cette collaboration artistique avec le trio orientalo-jazz Zyriab a donné lieu à la lecturespectacle MaQaMat مقامات (en 2006, reprise lors du Printemps des Poètes à Lyon, en 2012) et au spectacle musical et visuel Embrasure, ici loin d'ici [Centre Culturel Maison du Peuple de PierreBénite, 2011, avec la contribution de J. Tateossian (scénographie), F. Batt (réalisations plastiques), L. Palun (vidéaste), C. Allègre (sonorisation) et Cl. Deleuze (lumière)].

8. M. El Amraoui a participé à trois albums de Brain Damage : Spoken dub manifesto (Bangarang/ Jarring Effects, 2006); Short cuts (Jarring Effects/Discograph, 2008) et Empire Soldiers - Brain Damage \& Vibronics (Jarring Effects, 2013).

9. M. El Amraoui a notamment écrit des chansons pour le compositeur A. Salameh et la chanteuse N. Azzouz (Rissala, éd. Enja, Munich, Allemagne, 2006).

10. M. El Amraoui, A. Birot et M. Spitz, 2003, Tessons, Lario.

11. M. El Amraoui, 2007, Récits, partitions et photographies, La Passe du vent.

12. La quatrième de couverture de Récits, partitions et photographies (ouvr. cité) justifie la structuration du recueil selon «[...] une même préoccupation: dire la complexité des lignes intérieures et celle d'une certaine géométrie urbaine dont les lignes aussi se recoupent, se chevauchent ou s'enchevêtrent, comme la photographie d'un pont, le souffle d'une flûte, les gestes d'un mime, les bouts d'un journal intime, la rue et sa langue, la salle de bain, les informations par la télé ou la radio, la fête ou le lendemain d'une fête, le café, la gare... Toutes ces coïncidences qui, dans et par une poussée narrative, voudraient mesurer secrètement la fuite de leur sens, la surmonter. "

13. E. Morin, 2005, Introduction à la pensée complexe [1990], Paris, Seuil.

14. M. El Amraoui, De ce côté-ci alentour, ouvr. cité, p. 29.

15. Ibid., p. 30. 


\section{RÉSUMÉS}

Lors d'un entretien du 9 novembre 2017, Mohammed El Amraoui évoque son itinéraire de poètetraducteur en arabe et en français, au carrefour de plusieurs traditions poétiques - marocaine, française, mais aussi palestinienne, américaine ou grecque. Réfléchissant à la spécificité de chaque langue et à la complexité de leurs rapports, l'écrivain distingue l'acte traductif (qui opère le passage d'une langue à l'autre) de l'écriture bilingue (une pratique parallèle des deux langues). Le poète confie affronter l'« intraduisible radical » : en écrivant ses poèmes sans en anticiper la traduction, en s'immergeant dans les propriétés sémantiques, grammaticales et tout autant sonores, musicales d'une langue, pour ensuite tenter de s'autotraduire. Il revient également sur son travail avec d'autres poètes (Anas Alaili qu'il traduit) et avec la traductrice Catherine Charruau, avec des musiciens et des plasticiens : autant de collaborations qui interrogent la langue que se forge le poète, la singularité de sa forme et sa part de mystère.

During an interview on 9 November 2017, Mohammed El Amraoui evoked his journey as a poettranslator in Arabic and French, at the crossroads of several poetic traditions - Moroccan, French, but also Palestinian, American and Greek. Reflecting on the specificity of each language and the complexity of their relationships, the writer distinguishes between the act of translation (which involves the transition from one language to another) and bilingual writing (a parallel practice of the two languages). The poet confides in confronting the "untranslatable radical": by writing his poems without anticipating their translation, by immersing himself in the semantic, grammatical and equally sonorous and musical properties of a language, and then trying to translate himself. He also reviews his work with other poets (Anas Alaili whom he translates) and with translator Catherine Charruau, with musicians and visual artists: all collaborations that question the poet's language, the singularity of his form and his element of mystery. 